\title{
ANÁLISIS DEL EQUILIBRIO ENTRE EL POTENCIAL TURÍSTICO Y LA OFERTA DE ALOJAMIENTOS EN TURISMO RURAL MEDIANTE TÉCNICAS DE ESTADÍSTICA ESPACIAL. UNA APLICACIÓN A LA PROVINCIA DE CÁCERES (ESPAÑA) ${ }^{1}$
}

\author{
José Manuel Sánchez Martín* \\ Marcelino Sánchez Rivero** \\ Juan Ignacio Rengifo Gallego ${ }^{* * *}$ \\ Universidad de Extremadura
}

\section{RESUMEN}

La distribución de la oferta de alojamiento rural se concentra en espacios muy concretos coincidentes, en buena parte de los casos, con la presencia de los atractivos naturales más relevantes. Entre ellos destacan el relieve y la red hidrográfica como elementos preferidos por la demanda. No obstante, parte de la oferta alojativa se localiza en áreas con menos aptitudes para desarrollar esta modalidad turística. Por ello, proponemos realizar un análisis que nos permita determinar la bondad de la adecuación entre la oferta y el atractivo turístico mediante la utilización de estadística espacial. En concreto, se propone utilizar la regresión geográficamente ponderada y su modelo de ajuste para determinar si los alojamientos se localizan en las zonas idóneas o, por el contrario, están en áreas menos adecuadas para la práctica del turismo rural.

Palabras clave: Regresión geográficamente ponderada, I de Moran, regresión de mínimos cuadrados, evaluación del potencial turístico, Cáceres.

Recibido: 15 de julio de 2015

Devuelto para su revisión: 5 de febrero de 2016

Aceptado: 6 de abril de 2016

* Facultad de Empresa, Finanzas y Turismo. Avda. de la Universidad s/n. 10003 CÁCERES (España). E-mail: jmsanche@unex.es

** Facultad de Ciencias Económicas y Empresariales. Avda. de Elvas, 06071 BADAJOZ (España). E-mail: sanriver@unex.es

*** Facultad de Filosofía y Letras. Campus Universitario, s/n. 10003 CÁCERES (España). E-mail: irengifo@unex.es

1 Este artículo se encuadra dentro de las investigaciones llevadas a cabo durante la ejecución del Proyecto "Análisis y Planificación del turismo rural en la provincia de Cáceres" (PRI-IB 10092) subvencionado por la Dirección General de modernización e innovación tecnológica de la Junta de Extremadura. Fondos FEDER (Unión Europea). 


\title{
Analysis of the balance between tourist potential and supply of rural tourism accommodation using spatial statistical techniques. An application to the province of Cáceres (Spain)
}

\begin{abstract}
The distribution of the supply of rural accommodation is concentrated in very specific areas that coincide, in most cases, with the presence of the most outstanding natural attractions. Among them, landscape and the hydrographic network have been emphasized as preferred elements by the demand. However, part of the accommodation supply is located in areas with less aptitude to develop this form of tourism. Therefore, we propose an analysis that allows us to determine the goodness of fit between supply and tourism appeal using spatial statistics. Specifically, we propose using geographically weighted regression and adjustment model to determine whether accommodation is located in suitable areas or, on the contrary, in areas less suitable for the practice of rural tourism.
\end{abstract}

Keywords: geographically weighted regression (GWR), Moran's I, least squares regression, assessment of tourism potential, Cáceres.

\section{INTRODUCCIÓN}

Durante la década de los 60 del siglo pasado se produjeron en España una serie de cambios generales y específicos que contribuyeron al impulso de las actividades recreativas, principalmente en los espacios de litoral. El acceso generalizado a la motorización, la mejora de accesibilidad aérea y terrestre y, por supuesto, la disposición de un mayor volumen de rentas, propiciaron que, poco a poco, los espacios rurales de interior fueran vistos como lugares en los que se podían desarrollar actividades recreativas. No obstante, no será hasta entrado el decenio de los 70 cuando aparezcan líneas de investigación que adquieran cierta solidez a la hora de hablar del uso recreativo de los espacios rurales (López y Mulero, 1997). Por este motivo, se puede señalar que a partir de este periodo se observa la consolidación de tímidos flujos turísticos que se desplazan hacia el medio rural, como consecuencia del interés que despiertan algunos de sus recursos más valorados: balnearios, nieve, caza, pesca, espacios naturales o pequeños conjuntos históricos protegidos. Este interés se vio reflejado, con el paso del tiempo, en el crecimiento del número de alojamientos de diferentes tipologías a nivel nacional hasta que, en la década de los 80 y 90, las comunidades autónomas comienzan a regular y promocionar intensamente el sector del turismo rural.

El notable interés que despertó en su día el turismo rural se debe al papel que puede desempeñar en el incremento de rentas en estos espacios, máxime cuando la rentabilidad de las explotaciones agrarias y ganaderas ha descendido de forma notable a lo largo de los últimos años.

Esta idea primigenia ha tenido una repercusión muy desigual en el contexto español debido a la diferente vocación, tanto turística como económica, de su realidad provin- 
cial. Así, es posible hablar de zonas fuertemente "turistificadas" o de zonas donde la agricultura es escasa, poco rentable y poco competitiva y en las que el turismo rural puede ser un inicio de diversificación económica, de promoción de su territorio y de freno a su despoblación (Cànoves et al, 2004). A pesar de ello, también se detectan alojamientos rurales en entornos naturales de gran belleza y singularidad, si bien, durante la mayor parte del año, se observa que los alojamientos rurales disponen de un porcentaje de ocupación reducido, algo que puede explicarse no solo por la situación económica adversa, sino por una sobreoferta, una localización poco acertada, una meteorología poco propicia, una inadecuada o insuficiente promoción, etc.

Considerando dicha situación se propone utilizar modelos estadísticos basados en la parametrización espacial, de tal forma que combinen los aspectos más destacados del potencial turístico con el volumen de plazas de alojamiento rural existente en todo el territorio, lo que permitirá conocer el grado de adaptación entre sendos aspectos.

La actividad turística se ha analizado profusamente desde una óptica estadística tal como lo demuestra la enorme variedad de estudios que, desde diferentes perspectivas, aplican diversos análisis a través de este conjunto de técnicas sobre la mayor parte de variables e indicadores del turismo. Entre todos ellos destaca el análisis simultáneo sobre múltiples variables aplicando técnicas como el análisis factorial, ya sea exploratorio ya sea confirmatorio, el análisis clúster, etc. (San Martín et al, 2006; López Fernández et al, 2007; Brida et al, 2012; Álvarez García et al, 2012; Merinero et al, 2014). Asimismo, son destacables los estudios que se centran en la utilización de los parámetros estadísticos descriptivos enfocados normalmente al análisis de la oferta. Mientras tanto, el análisis inferencial suele aplicarse a análisis de demanda, en los que cobra especial importancia la predicción, no solo del número global de visitantes en futuras temporadas, sino del vector de características de cada uno de los turistas venideros, para una mejor adecuación entre la oferta y la demanda (Hernández, 2002).

Pese a estas tres aplicaciones diferentes y contrastadas del análisis estadístico al sector del turismo, conviene reseñar que desde hace más de dos décadas, en los estudios empíricos, los investigadores buscan detectar posibles asociaciones entre las características regionales y los resultados observados en diferentes localizaciones geográficas (Powell, 1991; Mennis, 2005; Taylor, 2008; Yi-Ju Chen, 2012), aspecto que implica necesariamente la utilización de estadística espacial.

Este hecho es comprensible si se considera que el turismo, al igual que la mayor parte de las actividades económicas, tiene un marcado matiz territorial debido a que tanto la oferta como los atractivos se distribuyen sobre un territorio que conforma el espacio turístico. Esta distribución espacial en algunos componentes del medio que configuran el potencial turístico sigue, en buena parte de los casos, un patrón marcado. Esto se ha puesto de manifiesto en diferentes estudios (Sánchez, 2009, 2013; Rengifo, 2012) en los que se obtienen correlaciones lineales significativas entre la presencia de plazas en alojamientos rurales y variables como la altimetría, la red hidrográfica o el propio clima. En otros casos, aparece una dispersión interesante como se plasma en la existencia de recursos de carácter cultural.

Por esta peculiaridad, se cree que el análisis espacial debe desempeñar un papel primordial en cualquier estudio turístico, algo que puede ir mucho más allá de la simple 
geolocalización de los principales parámetros turísticos, sobre todo si se apuesta por un análisis estadístico de corte espacial. De esta forma se podría eliminar una de las grandes deficiencias actuales del análisis turístico como es no considerar en toda su dimensión el carácter espacial del mismo, ya que un destino turístico no puede ser analizado de manera aislada, sino que se deben tener en cuenta las influencias que los destinos próximos pueden ejercer sobre el mismo, o viceversa (Sánchez, 2008).

Tradicionalmente se ha utilizado un modelo de regresión lineal utilizando el método de los mínimos cuadrados ordinarios para explicar una variable dependiente partiendo de diversas variables independientes (Banerjee, Gelfand, Sirmans, 2003) suponiendo que son homogéneos en el espacio (Charlton, 1997), si bien es preciso señalar que en la práctica, la asociación entre sendos tipos de variables es diferente en el territorio y los resultados deben ser, asimismo, variables desde una óptica geográfica, tal como se pone de manifiesto en diferentes estudios (Jones, 1995; Fotheringham y Brunsdon, 1999; Brunsdon, Fotheringham y Charlton, 2002).

Al considerar esta diversidad de estudios se pone de manifiesto que la utilización de un indicador global, como puede ser la ecuación descrita por una regresión múltiple, puede encubrir una realidad que, de facto, se observa al superponer sobre el territorio las variables que pueden explicar la localización de la oferta. Esta limitación se ha tratado se solventar mediante el análisis de regresión geográficamente ponderada (GWR) utilizada profusamente en la literatura de la última década (Huang y Leung, 2002; Longley y Tobon, 2004, Mennis y Jordania, 2005; Chen, 2012).

Esta técnica construye, en nuestro caso, una ecuación distinta para cada una de las entidades territoriales incorporando las variables independientes que configuran el atractivo turístico y considerando como variable dependiente el volumen de plazas en alojamiento rural. Con ello se consigue una ecuación individualizada que permite, mediante la utilización de diferentes parámetros, ya sea del tipo núcleo, ancho de banda, distancia o cantidad de vecinos, medir la adecuación de la variable dependiente al modelo construido, obteniéndose además el desajuste entre el valor real y el calculado por el modelo.

Se puede considerar, por tanto, que la regresión espacialmente ponderada es una herramienta útil para nuestro propósito, si bien no está exenta de limitaciones, ya que dependiendo del modelo generado a partir de las variables independientes se puede incurrir en problemas de multicolinealidad, algo que a la postre implicaría la obtención de ecuaciones incorrectas y, consecuentemente, resultados erróneos, a no ser que se realicen las depuraciones pertinentes.

La investigación se estructura en cuatro partes bien diferenciadas. En la primera, se realiza un breve repaso por la literatura especializada en análisis estadístico de parámetros turísticos. En la segunda, se expone la metodología seguida, explicando el caso de estudio y las técnicas de análisis utilizadas. En la tercera, se describe el proceso seguido para la realización de la regresión geográficamente ponderada y se exponen los resultados. Por último, en la cuarta y última parte se establecen las conclusiones más relevantes sobre la investigación. 


\section{METODOLOGÍA}

\subsection{El caso de estudio}

La provincia de Cáceres, que a mediados de los 90 era un territorio periférico, fronterizo y alejado de los principales focos de atracción turística a nivel nacional, comienza a integrarse en el mercado turístico de forma lenta y progresiva, con la excepción de algunas comarcas montañosas del Norte que mostraron una aceleración superior.

Se observa en todo el territorio analizado una doble vocación turística y, consecuentemente, de espacios turísticos, hecho que precisa de un análisis diferenciado, ya que las áreas donde se desarrolla el turismo cultural suelen localizarse en poblaciones muy concretas, en las que la actividad turística se ha ido asentando en un periodo más dilatado. Mientras tanto, los espacios rurales, en los que el auténtico atractivo turístico es el paisaje en su sentido más amplio, han comenzado a desarrollar el turismo en tiempos más recientes.

\section{Figura 1 \\ ZONA DE ESTUDIO}

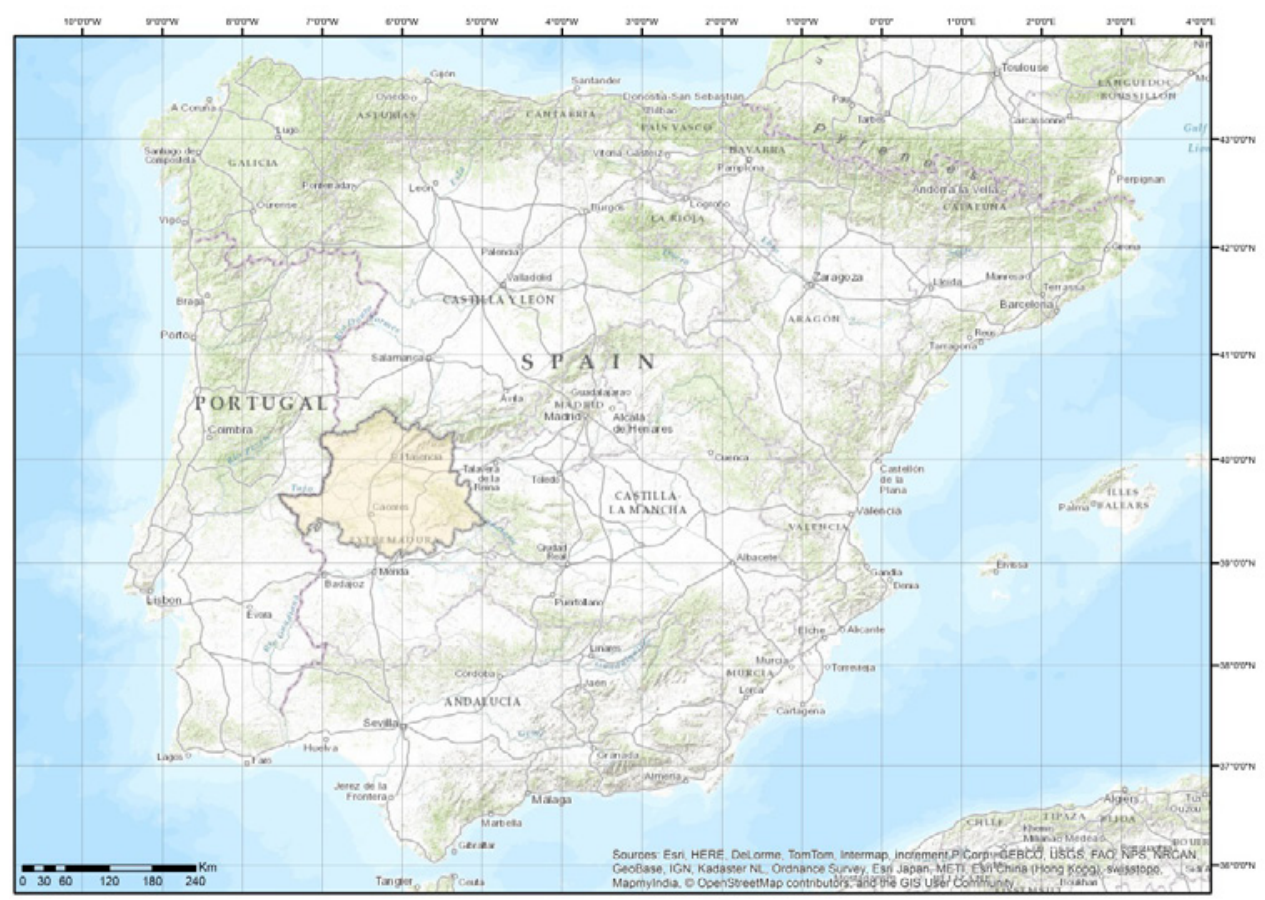

Este cercano desarrollo del turismo rural ha provocado que la implantación de la oferta se haya realizado de forma casi espontánea en numerosos municipios que tienen como característica común la montaña y otros atractivos vinculados a ella: agua, vegetación 
frondosa, etc. No menor importancia ha tenido el hecho de que esta fase de desarrollo expansivo haya coincidido con un periodo de bonanza económica (finales del siglo pasado y principios del actual) durante el cual el sector turístico experimentó un fuerte crecimiento en las áreas de interior. Esta situación ha desencadenado un aumento en la oferta de alojamientos rurales que afecta a numerosos núcleos de población, aunque no tuvieran los principales atractivos que demanda el turista rural, lo que pone de manifiesto una inadecuación entre el potencial turístico y la oferta de alojamientos presente en el territorio. Además, el crecimiento rápido y poco planificado favorece que no se haya puesto en marcha una política turística adecuada para suplir esta deficiencia de atractivos determinantes con la generación de productos turísticos dependientes de otros recursos presentes en la zona.

Sin embargo, dadas las vicisitudes actuales por las que atraviesa la economía del país, el turismo rural ha sido uno de los grandes perjudicados. Por este motivo, este artículo propone conocer la adecuación entre la oferta de alojamientos rurales y el potencial para el desarrollo de esta modalidad de turismo.

El aumento en la oferta de alojamientos rurales de la provincia ha sido muy notable a lo largo de los últimos años, como se corrobora en los datos publicados por la Encuesta de Ocupación en Alojamientos de Turismo Rural (EOTR) del Instituto Nacional de Estadística para el periodo 2005-2014. Según esta fuente oficial, el incremento se cifra en un $240 \%$ respecto del volumen de plazas existente en 2005.

Esta situación requiere un análisis específico para determinar su adaptación a la presencia de recursos turísticos de interés. Por ese motivo se parte de su localización, comparándola con el potencial existente para la práctica de esta modalidad turística.

En líneas generales, su distribución sobre el territorio se adapta a un potencial turístico variable, determinado previamente por otros estudios referidos a su medición (Rengifo, 2012; Sánchez, 2013). El grado de ajuste sigue en determinadas partes del territorio un patrón de distribución bastante acertado, si bien en otros espacios el nivel de adecuación entre sendos aspectos no es el idóneo. A ello se debe añadir que el aumento en el volumen de plazas no se ha correspondido con un crecimiento tan significativo en el número de turistas y pernoctaciones según se deduce de los datos publicados por el Instituto Nacional de Estadística (INE), ya que para ese mismo periodo se pasa de 65.000 turistas a 113.000 , y de 135.000 pernoctaciones a tan solo 242.000. Es decir, en ninguna de las dos variables se duplica la demanda, algo que sí ha sucedido con las plazas de alojamiento rural.

Si se considera el cociente entre las pernoctaciones y las plazas, la situación se torna aún más problemática, debido a que si en 2007 se alcanzaban casi 60 pernoctaciones al año por cada plaza de alojamiento disponible, en 2013 ni siquiera se consiguen 39, en 2014 apenas supera las 44. Es decir, para mantener las mismas proporciones que en 2007 a lo largo de 2014 tendrían que haberse registrado unas 380.000 pernoctaciones, muy distantes de las 242.000 reales que se contabilizaron.

Este desequilibrio entre oferta y demanda se ha puesto de manifiesto de una forma más aguda durante la actual crisis económica, hecho que obliga a plantearse si realmente existe una adecuación entre sendos aspectos vinculados al potencial turístico que reúne cada uno de los núcleos de la provincia, así como a detectar los espacios donde el grado de adaptación puede ser mayor o menor. 
Tabla 1

EVOLUCIÓN DE LAS PRINCIPALES MAGNITUDES TURÍSTICAS DEL TURISMO RURAL EN LA PROVINCIA DE CÁCERES

\begin{tabular}{|c|c|c|c|c|}
\hline & Plazas & Turistas & Pernoctaciones & Pernoctaciones/ plazas \\
\hline 2005 & 2.668 & 65.508 & 134.781 & 50,5 \\
\hline 2006 & 3.599 & 94.359 & 210.330 & 58,4 \\
\hline 2007 & 4.313 & 114.157 & 255.351 & 59,2 \\
\hline 2008 & 4.886 & 101.363 & 243.175 & 49,8 \\
\hline 2009 & 5.517 & 107.114 & 249.625 & 45,2 \\
\hline 2010 & 5.496 & 97.262 & 233.399 & 42,5 \\
\hline 2011 & 5.561 & 102.521 & 245.823 & 44,2 \\
\hline 2012 & 6.042 & 104.175 & 241.446 & 40,0 \\
\hline 2013 & 6.181 & 96.063 & 240.310 & 38,9 \\
\hline 2014 & 6.408 & 112.979 & 242.198 & 44,3 \\
\hline
\end{tabular}

Fuente: www.ine.es

\section{Figura 2 \\ DISTRIBUCIÓN DE LOS ALOJAMIENTOS \\ RURALES EN LA PROVINCIA DE CÁCERES}

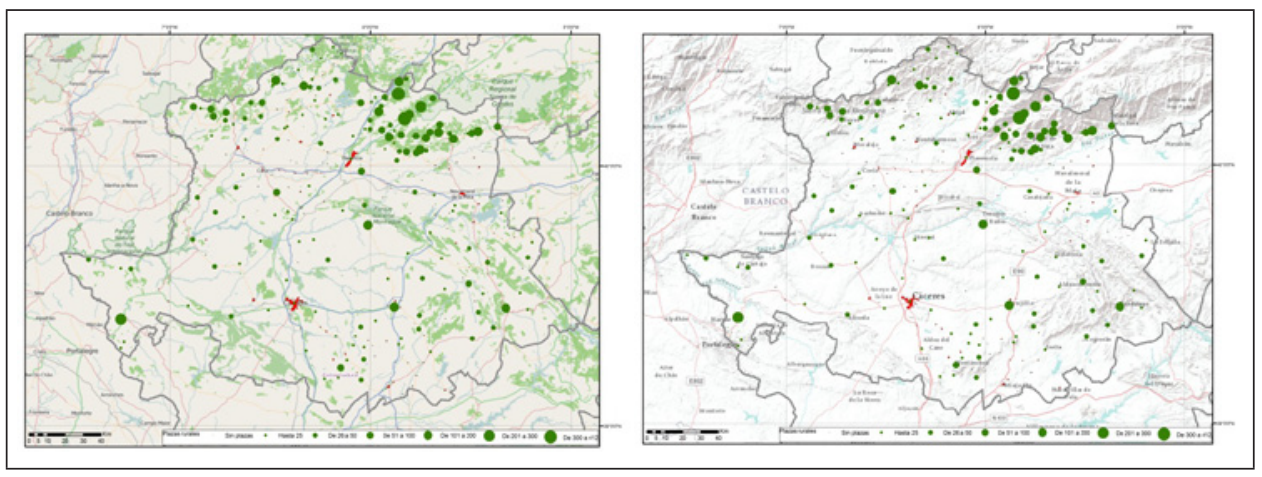

La localización predilecta para este tipo de oferta coincide mayoritariamente con dos atractivos clave: la proximidad a los relieves más elevados y la presencia de cursos de agua cristalina procedentes de las montañas, según se desprende de algunas investigaciones donde se obtenían correlaciones muy interesantes entre la presencia de alojamientos rurales y las zonas montañosas, independientemente de la existencia de otros recursos vinculados en mayor o menor medida al turismo rural (Sánchez, 2009). La presencia de estos dos grandes atractivos se complementa con la aparición de infraestructuras turísticas muy concretas, 
como las piscinas naturales o zonas habilitadas para el baño, de gran interés para los turistas, aspecto éste que se pone de manifiesto cuando se analizan las preferencias de la demanda.

También cabe destacar que la implantación de alojamientos a veces ha sido sobrevalorada, pensando tal vez que la mejor opción para el desarrollo de la actividad era la instalación de alojamientos, sin pensar en la adecuación del potencial turístico a la oferta existente ni en la generación de productos turísticos diferenciados, aspecto éste que cobra especial relevancia cuando se detectan otros atractivos muy repartidos por el territorio como espacios naturales protegidos.

Según lo expuesto, se observan ciertos desajustes, ya que por una parte aparece una oferta de alojamientos rurales en entornos con un potencial limitado respecto de los principales atractivos para la demanda y, por otra, se detecta que en los espacios de mayor potencial, la oferta sigue aumentando, pese a que los porcentajes de ocupación no son los mejores para asegurar un rendimiento óptimo de la actividad.

Esta casuística, unida a los efectos devastadores derivados de la profunda recesión económica que afecta al país estos últimos años, pone en peligro el mantenimiento de algunos alojamientos, dado que durante la fase expansiva de la actividad, la planificación era deficiente. Pese a ello, es preciso destacar que el aumento de alojamientos rurales redunda en beneficio de la actividad turística, ya que si no se cuenta con una oferta adecuada, variada y de calidad, de nada o casi nada sirve disponer de espacios idóneos para la práctica de esta modalidad turística.

\subsection{Técnicas de análisis}

El análisis estadístico posee una enorme complejidad causada no solo por el grado de dificultad que caracteriza al sistema turístico, sino también por los problemas de aplicación que tienen las técnicas multivariantes. Sendos inconvenientes se acentúan cuando se propone la utilización de una técnica tan complicada como la regresión geográficamente ponderada, muy novedosa en los análisis territoriales del turismo, lo que entraña no pocas limitaciones a la hora de seguir las pautas propuestas por otros autores. Al mismo tiempo, cabe destacar que este estadístico está condicionado por numerosos aspectos, muchos de los cuales inciden de forma directa en la bondad de los resultados obtenidos.

Este tipo de regresión (Sánchez, 2012) estima un modelo lineal que queda definido por:

$$
y_{i}=B_{0}(i)+B_{1}(i) x_{1 i}+B_{2}(i) x_{2 i}+B_{n}(i) x_{n i}+E(i)
$$

siendo la localidad en la que se miden las variables independientes, además de la dependiente para la cual se estiman los parámetros. Éstos se obtienen de:

$$
B^{\prime}=\left(X^{T} W(i) X\right)^{-1} X^{T} W(I) Y
$$

donde $W(I)$ es la matriz de pesos específicos para la ubicación $i$, de tal forma que las observaciones cercanas tienen más peso que las lejanas. 
El hecho de concebir el turismo como una actividad íntimamente vinculada a un territorio ha motivado que se apueste por la aplicación de la regresión ponderada espacialmente (GWR), estando su elección justificada porque es una técnica que permite elaborar modelos inferenciales que tienen en las relaciones espaciales una de sus fortalezas principales y que, a su vez, la diferencia de otro tipo de regresiones múltiples.

Esta técnica proporciona un modelo local de la variable dependiente partiendo de las independientes. Calcula, por consiguiente, una ecuación para cada entidad analizada, al contrario de lo que sucede con otro tipo de regresiones, en los que se obtiene un modelo global. La bondad de esta técnica se ha puesto de manifiesto en numerosos estudios, vinculados a diferentes aplicaciones y siguiendo variados métodos de obtención (Wheeler y Tiefelsdorf, 2005; Duque et al, 2011; Chen et al, 2012). Supone un avance notable sobre otras regresiones, pues permite ir de una visión global a un análisis local del problema, aportando mayor grado de detalle (Lloyd y Shuttleworth, 2005).

El análisis de este tipo de regresión se realiza considerando las diferentes posibilidades de configuración analítica que permiten las herramientas de modelado espacial del software ArcGIS. Este programa permite decidir el ajuste de los parámetros de núcleo (fijo o adaptativo), ancho de banda (criterio de información Akaike, validación cruzada o ancho de banda fijo), distancia o cantidad de vecinos. Cada uno de ellos presenta variaciones, de mayor o menor calado, sobre los resultados que ofrece el modelo diseñado, por lo que es preciso tomar ciertas decisiones para afrontar los cálculos con seguridad.

Pese a que este tipo de regresión aporta el criterio espacial como elemento destacado y diferenciado respecto de otras regresiones, no se puede olvidar que está sometido a la problemática inherente a buena parte de las técnicas estadísticas complejas. Entre los principales inconvenientes que plantea su uso destacan la omisión de variables explicativas, la existencia de relaciones no estrictamente lineales, la presencia de observaciones atípicas y, sobre todo, la multicolinealidad, siendo este último uno de los inconvenientes más graves a los que se enfrenta un modelo de regresión.

Buena parte de dichos impedimentos puede ser subsanado mediante un análisis pormenorizado de la base de datos utilizada y a través de un conocimiento exhaustivo del sistema turístico que se analiza. En cambio, eliminar la redundancia de las variables explicativas resulta bastante más complejo puesto que en la mayor parte de los casos se produce por un error en la configuración del modelo de regresores. Dicha circunstancia puede invalidar la aplicación de ésta y cualquier otra regresión.

El origen de la colinealidad se debe a que una de las hipótesis del modelo de regresión lineal múltiple establece como conditio sine qua non que no exista relación lineal exacta entre los predictores, es decir, implica que no existe una correlación perfecta en el modelo. Este escenario es infrecuente en la realidad, si bien aparece muy a menudo una multicolinealidad imperfecta, debido tanto a la relación entre variables independientes como a deficiencias en la información de base (Uriel, 1990).

Las interrelaciones parciales entre las variables, que se ponen de manifiesto con la multicolinealidad imperfecta, son frecuentes en los modelos turísticos, ya que la interacción que se establece entre algunos recursos turísticos es muy fuerte, como sucede por ejemplo con la configuración y tipología del relieve, el clima, la hidrografía, la vegetación, o la población y equipamientos turísticos. En este sentido cabe mencionar la fuerte correlación 
que existe cuando se analizan de forma conjunta variables como la altitud y la temperatura o el tipo de clima, muy condicionados por el factor climático, que también actúa como elemento atractor dentro del sistema que configura el turismo rural. Esta situación descrita, pese a no tener una correlación perfecta, sí que supondría obtener unos coeficientes elevados, impidiendo por ejemplo que la altitud y el tipo de clima actuasen como variables independientes de forma simultánea.

Para solventar en la medida de lo posible estas deficiencias, inherentes al diseño de modelos complejos, no existen técnicas estadísticas especialmente diseñadas para ello. No obstante, si se quiere eliminar buena parte de la colinealidad, se puede optar por utilizar ya sea el conocimiento teórico del sistema turístico analizado, ya sean ciertos cálculos estadísticos que posibiliten determinar cómo afecta este problema a la estimación obtenida respecto al modelo. Entre estos últimos destacan la matriz de correlación lineal, el factor de inflación de la varianza (Uriel, 1990) e incluso análisis de componentes principales. Conviene reseñar asimismo que existe cierta incertidumbre sobre la utilización de estas técnicas ya que, en ocasiones, la elevada correlación puede deberse a casualidad y no a causalidad (Sánchez, 1995). Por todo ello, según se desprende de la literatura estadística, resulta difícil detectar la presencia de multicolinealidad, si bien, en la práctica se suele utilizar el número de condición como elemento discriminador (Rachudel, 1971; Belsley, 1980, 1982).

\section{RESULTADOS}

La actividad turística tiene como escenario privilegiado el espacio geográfico en el que se desarrolla y está condicionada, en buena parte de los casos, por una serie de factores que tienen cierta continuidad espacial. Los recursos turísticos presentes en el territorio pueden afectar no solo a un núcleo de población, sino que lejos de ello, puede abarcar a varios, de donde se deduce que la proximidad puede incidir directamente en la configuración del espacio geográfico.

Tras valorar las ventajas e inconvenientes de diferentes técnicas que permiten detectar las fuertes correlaciones entre las variables predictoras, se ha optado por realizar diferentes regresiones exploratorias para conseguir un modelo adecuado y compararlo con otro definido por los autores. En la configuración original del modelo se combinan 19 parámetros con los que se persigue explicar la distribución de las plazas de alojamiento.

Con el conjunto de variables disponibles, se han obtenido 3.895 modelos diferentes de regresiones exploratorias, la mayor parte de las cuales no se ajustaban a los parámetros que se recomienda en la literatura específica. Por ello, se recurre a un factor de inflación de la varianza (VIF) que no sobrepase 7,5, considerado como límite aceptable cuando se trata de asegurar inexistencia de colinealidad; a la vez, se requiere que la autocorrelación espacial de los residuos obtenidos fuera inferior a 0,1 garantizando así su aleatoriedad. Como consecuencia de ello, se han seleccionado diferentes modelos para los que se han tenido en cuenta criterios como el mayor grado de ajuste y explicación, un factor de inflación de la varianza reducido y que presenten residuos aleatorios. Se caracterizan por disponer de 5 a 7 regresores, tanto para los obtenidos mediante regresión exploratoria como para el modelo diseñado. 


\section{Tabla 2 \\ CONFIGURACIÓN DEL MODELO DE REGRESIÓN DEL TURISMO RURAL EN LA PROVINCIA DE CÁCERES}

\begin{tabular}{|l|l|}
\hline Variables independientes & Confort térmico relativo \\
\hline Categoría del recurso natural & Población (nomenclátor 2012) \\
\hline Red hidrográfica & Rutas senderistas publicadas (FEMEX) \\
\hline Altimetría (radio $10 \mathrm{Km})$ & Periodo de visitas óptimo \\
\hline Vías pecuarias & Recursos Culturales $(20 \mathrm{Km})$ \\
\hline Embalses (radio $10 \mathrm{Km})$ & Empresas de actividades \\
\hline Atractivo turístico (pairwise) & Accesibilidad al municipio \\
\hline Biodiversidad (n ${ }^{\circ}$ de especies) & Demanda que puede atraer \\
\hline Singularidad & Información / Centros interpretación \\
\hline Complementos & \\
\hline Flora & \multicolumn{2}{|l}{} \\
\hline Variable dependiente & \\
\hline Plazas de alojamiento rural &
\end{tabular}

La mayor parte de los análisis exploratorios válidos coinciden en ciertas variables, como la altimetría, vías pecuarias, biodiversidad, empresas de actividades y centros de interpretación. Esta situación se corrobora en la mayor parte de las variables cuando se recurre al porcentaje de significancia que tienen las mismas. Así, destacan las empresas de actividades, con un $100 \%$ de significancia; las oficinas de información y centros de interpretación, a la que se unen las vías pecuarias, ambas con un 97\%; mientras tanto, las rutas senderistas, la biodiversidad y la altimetría, tienen valores de $94 \%, 92 \%$ y $81 \%$, respectivamente.

Por su parte, los resultados ofrecidos por la regresión de mínimos cuadrados ordinarios al utilizar el modelo desarrollado por los autores basado en el conocimiento empírico del sistema turístico del entorno analizado y de las opiniones vertidas por turistas, configuran un modelo algo diferente, con menor explicación de la varianza, pero tal vez más ajustado a la realidad.

Un somero análisis de la situación que arrojan los modelos de regresión más representativos (RE1 a RE4), nos da a entender que existen marcados atractores turísticos, que actúan como auténticos regresores a la hora de explicar la presencia de plazas de alojamiento rural (altimetría, biodiversidad), a la vez que existen otras variables independientes que pueden ser consecuencia del desarrollo de esta actividad (empresas de actividades y oficinas de información y centros de interpretación), pero sirven para matizar el volumen de plazas disponibles o el nivel de desarrollo alcanzado. Por otra parte, aparecen diferentes variables independientes que pueden servir de base para fomentar el desarrollo del turismo rural y sus actividades complementarias, como sucede en el caso de las vías pecuarias y rutas senderistas, pero que en ningún momento actúan como factores que condicionen el desarrollo del turismo rural, ya que en el mejor de los casos, las rutas senderistas atraen a excursionistas y, por tanto, no pernoctan en los alojamientos. Además, la variable población aparece en algunos modelos de regresión exploratoria como si fuera un factor 
de interés, si bien, ateniéndonos a la legislación vigente en esta materia, es simplemente una conceptualización del ámbito rural, algo que no impide que en cualquier territorio no urbano pueda instalarse un alojamiento rural. (DECRETO 65/2015, de 14 de abril, por el que se establece la ordenación y sistema de clasificación de los alojamientos de turismo rural de la Comunidad Autónoma de Extremadura, DOE 74, de 14 de abril de 2015).

Tabla 3

RESUMEN DE MODELOS MÁS VIABLES DE REGRESIÓN EXPLORATORIA

\begin{tabular}{|c|c|c|c|c|c|}
\hline Modelos & RE1 & RE2 & RE3 & RE4 & REC \\
\hline X1 & Altimetría & Altimetría & Altimetría & Altimetría & Altimetría \\
\hline X2 & Vías pecuarias & Vías pecuarias & Vías pecuarias & Vías pecuarias & $\begin{array}{c}\text { Red } \\
\text { hidrográfica }\end{array}$ \\
\hline X3 & Biodiversidad & Biodiversidad & $\begin{array}{c}\text { Rutas } \\
\text { Rutas } \\
\text { senderistas }\end{array}$ & $\begin{array}{c}\text { Rutas } \\
\text { senderistas }\end{array}$ & $\begin{array}{c}\text { Rutas } \\
\text { senderistas }\end{array}$ \\
\hline X5 & Población & Confort térmico & Población & Complementos \\
\hline X6 & $\begin{array}{c}\text { Información } \\
\text { / Centros } \\
\text { interpretación }\end{array}$ & $\begin{array}{c}\text { Información } \\
\text { / Centros } \\
\text { interpretación }\end{array}$ & $\begin{array}{c}\text { Información } \\
\text { / Centros } \\
\text { interpretación }\end{array}$ & $\begin{array}{c}\text { Información } \\
\text { / Centros } \\
\text { interpretación }\end{array}$ & $\begin{array}{c}\text { Información } \\
\text { / Centros } \\
\text { interpretación }\end{array}$ \\
\hline X7 & $\begin{array}{c}\text { Empresas de } \\
\text { actividades }\end{array}$ & $\begin{array}{c}\text { Empresas de } \\
\text { actividades }\end{array}$ & $\begin{array}{c}\text { Empresas de } \\
\text { actividades }\end{array}$ & $\begin{array}{c}\text { Empresas de } \\
\text { actividades }\end{array}$ & $\begin{array}{c}\text { Empresas de } \\
\text { actividades }\end{array}$ \\
\hline Adj2 & 0,41 & 0,40 & 0,40 & 0,39 & 0,38 \\
\hline MaxVIF & 2,02 & 1,99 & 2,04 & 1,36 & 1,76 \\
\hline SA & 0,08 & 0,04 & 0,08 & 0,05 & 0,08 \\
\hline
\end{tabular}

RE: Modelos de regresión exploratoria / REC: Modelo de regresión propuesto.

En cambio, el modelo basado en el conocimiento empírico del turismo rural de la zona analizada y en las opiniones de la demanda (REC) presenta una ligera disminución en la explicación de la varianza $(0,38$ frente a 0,41$)$, si bien puede ser más operativo y acertado a la postre, ya que utiliza factores que motivan los viajes turísticos $\mathrm{y}$, por lo tanto, las estancias en alojamiento. Se trata, a nuestro juicio, de un modelo robusto que permitirá afrontar con total garantía la aplicación de la regresión espacialmente ponderada, siendo el modelo que se utilizará para la aplicación de la regresión geográficamente ponderada.

La explicación de la varianza total reflejada por la regresión de mínimos cuadrados ordinarios que se obtiene con el mismo puede parecer limitada en un principio, si bien se deben entender las limitaciones que ofrece la propia variable dependiente, la localización y el volumen de plazas de alojamiento rural. Esta restricción está ocasionada por el hecho de que no siempre se han construido estos alojamientos en los lugares más indicados para tal fin, aquéllos en los que el potencial turístico así lo aconsejaba. Lejos de ello, hay establecimientos de esta tipología asentados en lugares que no cumplen con los principales requisitos que exige la demanda, lo que da lugar a la aparición de ciertos desajustes que condicionan el modelo. Estos establecimientos, lejos de ser considerados como outliers, forman parte de nuestro modelo, puesto que su supresión contribuiría a modificar la realidad. Al mismo tiempo, 
también se observa que hay lugares con un atractivo interesante para la práctica de esta modalidad de turismo, pero o bien carecen de alojamientos, o bien disponen de pocas plazas.

Los resultados detallados de la regresión de mínimos cuadrados ordinarios reflejado en la Tabla 4, muestran una situación alentadora sobre el modelo seleccionado, ya que los coeficientes (Coef) son elevados en la mayor parte de las variables, destacando cómo las empresas de actividades y la información turística y centros de interpretación tienen un peso fundamental, no se puede olvidar que tratan de prestar servicios a los turistas rurales. A estos coeficientes se añaden otros que reflejan la bondad en la configuración de nuestro modelo. Entre ellos se encuentra la prueba estadística T, utilizada para evaluar si una variable explicativa es estadísticamente significativa o no. Si la hipótesis nula es que el coeficiente, en todos los casos, es igual a cero, no ayuda al modelo y, por lo tanto, la variable en cuestión tendría que ser eliminada, circunstancia que como se observa no ocurre. Además, la probabilidad (Prob) y la probabilidad robusta (Robust) del presentan valores adecuados, destacando por último que el factor de inflación de la varianza (VIF) es reducido.

Tabla 4

RESULTADOS DEL MODELO Y DIAGNÓSTICO DE REGRESIÓN

\begin{tabular}{|c|c|c|c|c|c|}
\hline Variable & Coef & t_Stat & Prob & Robust & VIF \\
\hline Intercepción & $-94,43$ & $-8,57$ & 0,0000 & 0,00 & ------ \\
\hline Red hidrográfica & 2,75 & 1,14 & 0,25 & 0,29 & 1,42 \\
\hline Altimetría & 5,92 & 2,68 & 0,001 & 0,00 & 1,76 \\
\hline Biodiversidad & 7,64 & 2,47 & 0,01 & 0,00 & 1,49 \\
\hline Complementos & 3,21 & 1,89 & 0,06 & 0,07 & 1,21 \\
\hline Rutas senderistas publicadas & 9,53 & 3,43 & 0,00 & 0,01 & 1,30 \\
\hline Empresas de actividades & 26,19 & 6,75 & 0,00 & 0,01 & 1,11 \\
\hline Información / Centros Interpretación & 8,38 & 3,68 & 0,00 & 0,01 & 1,20 \\
\hline
\end{tabular}

Asimismo, una vez decidido el modelo de regresión que se utilizará, resulta conveniente recurrir al cálculo de la autocorrelación espacial I de Moran sobre los residuos obtenidos. Con ello se persigue verificar la aleatoriedad espacial de los mismos y, de ese modo, descartar la omisión de alguna variable predictora importante que pudiera tener una explicación de base territorial, a la vez que asegura que, efectivamente, se puede realizar una regresión espacial, pues resulta una condición imprescindible para la aplicación de esta técnica geoestadística.

La estructura de la I de Moran es la siguiente:

$$
I=\left(n / S_{0}\right) \sum_{i=1}^{n} \sum_{j=1}^{n} w_{i j} z_{i} z_{j} / \sum_{i=1}^{n} z_{i}^{2}
$$

donde $S_{0}=\sum_{i=1}^{n} \sum_{j=1}^{n} w_{i j}$ es la suma de elementos de la matriz de pesos. 
Las observaciones $z$ son las desviaciones de la media $\left(x_{i}-\bar{x}\right)$ o $\left(x_{j}-\bar{x}\right)$ donde $x_{i}$ es el valor de la variable en una unidad espacial determinada y $x_{j}$ es el valor de la variable en otra localización, normalmente las vecinas a $x_{i}$. Habitualmente, en la matriz, se les asigna el valor de 1 a los vecinos de cada unidad espacial y 0 al resto (Celemín, 2009).

Mediante la aplicación de esta técnica se busca la relación entre diferentes localizaciones y los valores que se alcanzan en la variable analizada, es decir, los residuos obtenidos mediante regresión de mínimos cuadrados, de tal manera que cuando los valores para las entidades vecinas son mayores que el promedio, el producto cruzado será positivo; mientras que cuando los primeros son inferiores al promedio y el valor de la entidad analizada es mayor, el producto cruzado será negativo. Así se revela si la variable analizada está agrupada, dispersa o es aleatoria. Aparte del índice sintético, se calcula una puntuación $z$ y un valor $p$, siendo la primera el valor de la desviación estándar, y la segunda es la probabilidad de que el patrón espacial observado se haya originado a través de algún proceso aleatorio, con lo que se confirmaría la hipótesis nula, es decir, la distribución aleatoria.

En nuestro estudio, dada la amplia gama de posibilidades que ofrece la autocorrelación espacial, se opta por aplicar la distancia euclidiana inversa como método de extracción.

Los resultados indican que la probabilidad de que la distribución sea aleatoria es muy elevada, condición indispensable para continuar con nuestro desarrollo.

\section{Figura 3 \\ GRÁFICO DE AUTOCORRELACIÓN ESPACIAL DE MORAN}

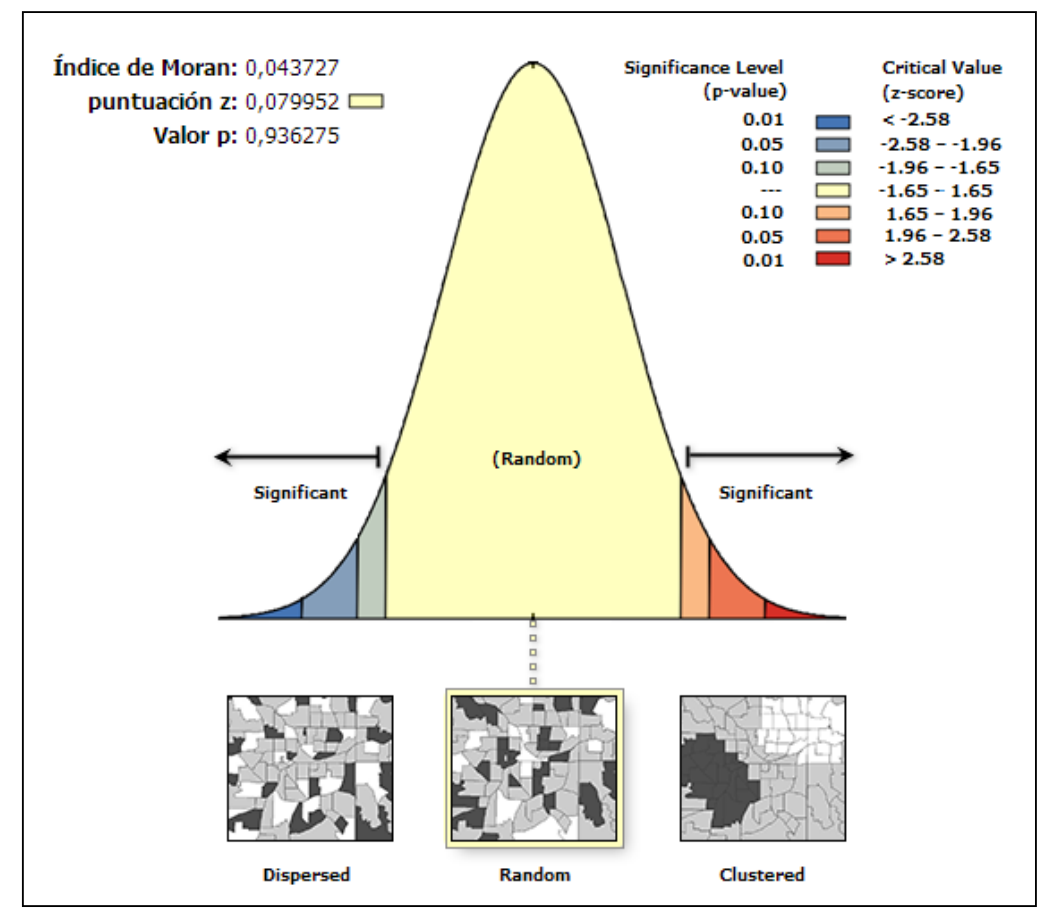


Una vez decidido el modelo de regresión y comprobada la aleatoriedad territorial que presentan los residuos obtenidos, es posible aplicar la regresión espacial, aunque para ello se precisa ajustar algunos parámetros. El primero que se debe fijar es si el núcleo de la función (kernel) toma una distancia fija o adaptativa, partiendo para ello de la propia distribución de los elementos tomados para el análisis. En el caso estudiado se toman como referencia los 320 núcleos (capitales de núcleo y entidades menores) que configuran la provincia de Cáceres, por lo que considerando tanto su número como su reparto sobre el territorio, podría parecer más indicado utilizar el kernel adaptativo. Sin embargo, cuando se utiliza este parámetro, tanto la distancia como el número de casos tomados como vecinos, se multiplica en exceso. A la vez, pese a tener un $\mathrm{R}^{2}$ elevado, cuando se procede a su ajuste, el descenso es notable $(0,67$ frente a 0,38 cuando se utiliza una distancia de $15 \mathrm{Km}$ ), por lo que se desecha esta opción para apostar por un kernel fijo. En este caso, las diferencias entre $\mathrm{R}^{2}$ y $\mathrm{R}^{2}$ ajustado son sensiblemente inferiores (0,53 y 0,48 respectivamente), indicativo de un modelo relacional más robusto.

Una vez decidido que el núcleo de la función que se utilizará es el fijo, se debe optar por el método que se utilizará para determinar la extensión del kernel. Entre las opciones disponibles figuran el criterio Akaike corregido, la validación cruzada y el ancho de banda determinado por el número de vecinos o la distancia. Tras probar las tres opciones se ha optado por aplicar el criterio de información Akaike corregido (AICc) sobre núcleos fijos. El fundamento de este criterio consiste en calcular una medida relativa de la bondad del ajuste obtenido mediante el modelo de regresión. Para ello se basa en la entropía del propio sistema concebido, es decir, el grado de desorden del mismo.

La formulación matemática de este criterio es la siguiente:

$$
A I C_{C}=A I C+\frac{2 k(k+1)}{n-k-1}
$$

siendo $n$ el número de datos de la muestra (320) y $k$ el número de parámetros (7) incluidos en el modelo estadístico.

La utilización del criterio de información AICc en vez de AIC se debe a que, pese a disponer de 320 entidades de análisis, puede que sea un volumen insuficiente para el cálculo de AIC, máxime si se considera que tan solo 154 disponen de plazas de alojamiento, a la vez que debe sopesarse la complejidad de un modelo con 7 predictores. En estos casos, en los que $n$ puede considerarse pequeño y/o $k$ grande, la literatura aconseja utilizar un parámetro de corrección sobre el criterio de información Akaike (Hurvich y Tsai, 1989; Burnham y Anderson, 2002).

Junto a ello, conviene reseñar que se ha asignado mayor peso en el modelo a los núcleos que disponen de un volumen de plazas superior, con lo que se reduciría la incertidumbre que puede existir ya que numerosos casos carecen de alojamientos rurales y, consecuentemente, el número de plazas es cero. 
El modelo propuesto refleja un factor de condición asumible, ya que en ningún caso se supera el umbral crítico de 30, concentrándose en la mayor parte de los casos en valores inferiores a 25, algo muy positivo cuando se cuenta con una muestra de 320 casos.

Los resultados que ofrece la técnica revelan un $\mathrm{R}^{2}$ del $72 \%$ frente a uno ajustado del $71 \%$, por lo que se entiende que el modelo desarrollado es robusto y tiene una contribución notable a la explicación de la varianza sobre las plazas en alojamientos rurales (Tabla 5 y Figura 4).

Tabla 5

RESULTADOS DE GWR

CON CRITERIO AKAIKE (AICC)

\begin{tabular}{|c|c|c|}
\hline & Valores & Definición \\
\hline AICc & 4535,55 & Criterio de información Akaike corregido \\
\hline $\mathrm{R}^{2}$ & 0,72 & \\
\hline $\mathrm{R}^{2}$ Ajustado & 0,71 & Plazas \\
\hline \multicolumn{2}{|c|}{ Variable dependiente } & Red hidrográfica \\
& Altimetría \\
& Biodiversidad \\
& Complementos \\
& Variables independientes & Rutas senderistas publicadas \\
& Empresas de actividades \\
& Información turística / Centros interpretación \\
\hline
\end{tabular}

A pesar de la bondad del modelo, resulta obvio que existe un desajuste entre los valores reales y observados de plazas de alojamiento rural, algo normal cuando se analiza un sistema complejo. Por ello, se precisa encontrar una explicación a este desajuste, más allá de la suficiencia estadística, pues al analizar diferentes magnitudes de residuales, se observan ciertas pautas comunes.

Con el fin de verificar el ajuste entre las plazas observadas y las predichas en cada uno de los casos analizados, se ha realizado una regresión lineal, cuyo ajuste es del 40\%, si bien lo más destacable es que los mayores desajustes se producen en aquellos núcleos que no disponen de alojamiento y de algunos casos, muy concretos, donde el volumen de plazas observado y calculado difiere de forma notable. Serán éstas las entidades que precisarán actuaciones correctoras mediante planes específicos vinculados a la generación de productos turísticos o a la diversificación de los mismos. 


\section{Figura 4}

\section{$R^{2}$ LOCAL DERIVADA DE GWR CON CRITERIO AICC}

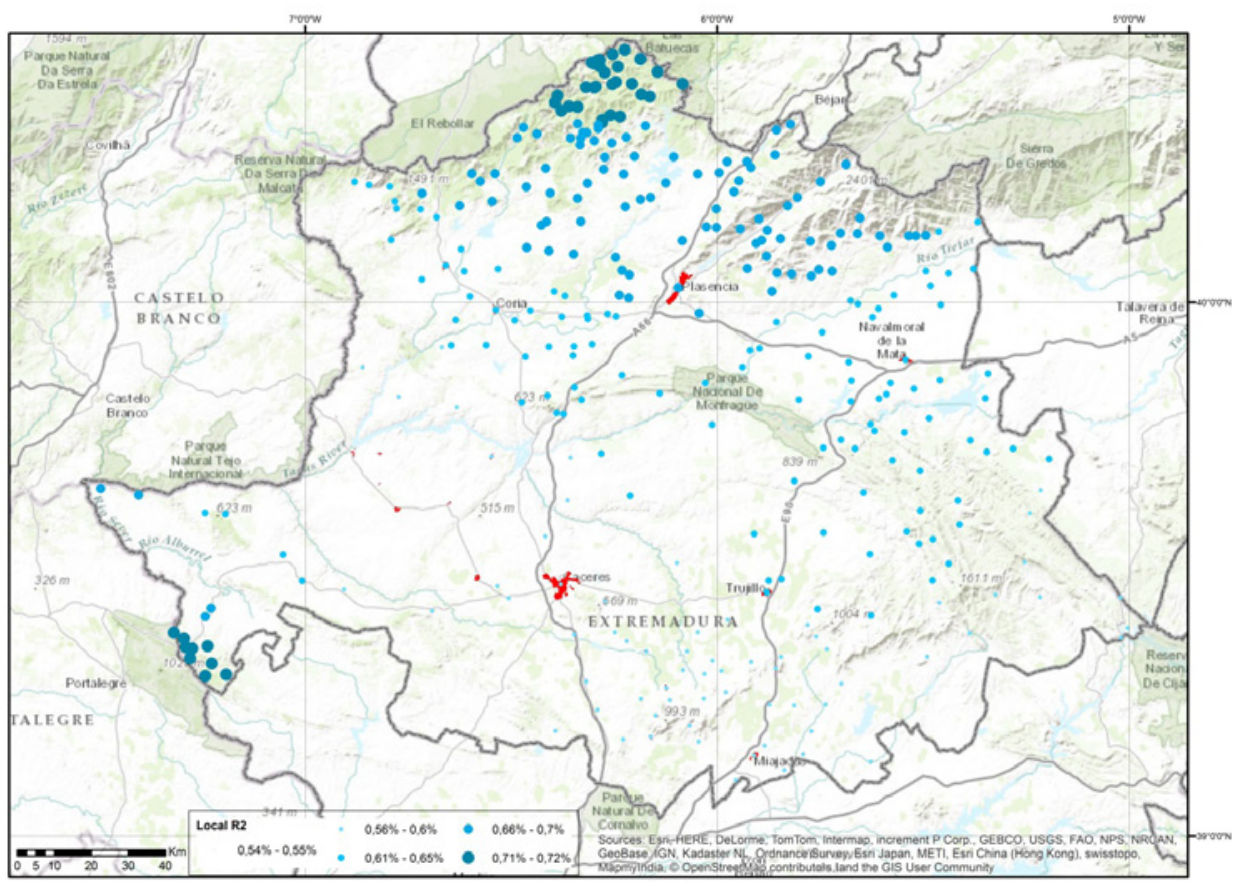

Figura 5

AJUSTE LINEAL ENTRE PLAZAS OBSERVADAS Y PREDICHAS

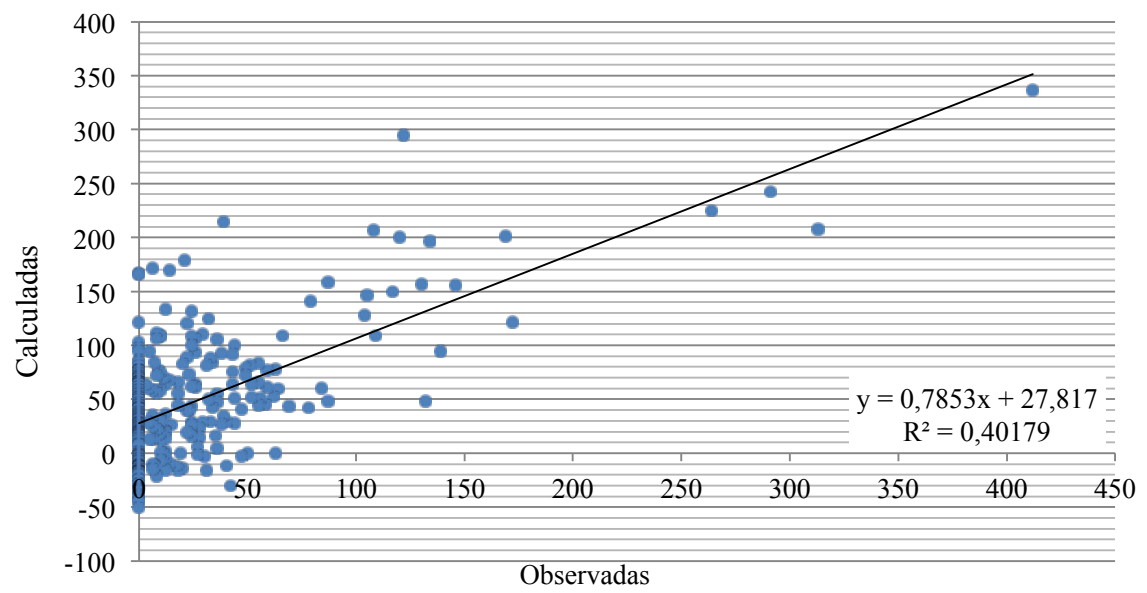


Existen dos causas probables que pueden provocar el desajuste:

- La no inclusión de variables explicativas, algo posible, si bien conviene destacar que se han descartado variables independientes debido a problemas de colinealidad e incluso incluyéndolas en el modelo no aumentaban de forma significativa la explicación. Como ya se apuntaba, incrementar la explicación mediante la inclusión de variables fuertemente correlacionadas presenta un problema metodológico grave, por lo que se optó solo por la inclusión de aquellas variables más significativas y carentes de este problema de interconexiones entre los predictores.

- El modelo detecta que son las plazas de alojamiento las que no se ajustan al auténtico potencial turístico del territorio, algo que cobra especial significación si se tiene en cuenta que hay núcleos en los que la concentración de plazas ha sido muy elevada, con más de 400; mientras tanto, también sucede que otros espacios, muy próximos y con un potencial turístico elevado, apenas disponen de alojamientos rurales. Esta situación se traduce en una pérdida de explicación en el modelo desarrollado al detectarse estos casos anómalos, algo que afecta a núcleos carentes de alojamiento rural, por diferentes motivos.

No obstante, los resultados detallados de la regresión espacial muestran un ajuste promediado entre todas las entidades del 64\%, cifra nada desdeñable, máxime si se considera que el factor de condición ofrece una media de 24,5, valor inferior al crítico, estimado en 30 puntos. Asimismo, la cifra media de los residuos es de $-23,2$ plazas. Pese a todo, esta situación promediada encubre variaciones (Tabla 6) cuando se recurre al cálculo de los parámetros de dispersión más usuales.

\section{Tabla 6 \\ MEDIDAS DE DISPERSIÓN SOBRE LOS RESULTADOS DE GWR (N= 320 ENTIDADES)}

\begin{tabular}{|c|c|c|c|c|c|c|c|}
\hline & Media & Mín. & Máx. & Cuartil 1 & Cuartil 2 & Cuartil 3 & Des. Est. \\
\hline Plazas Observadas & 22 & 0 & 412 & 0 & 0 & 26 & 45,7 \\
\hline Plazas Predichas & 45 & -50 & 337 & 0,77 & 36,3 & 69,6 & 56,7 \\
\hline Fact. Condición & 24,5 & 20,3 & 29,9 & 22,4 & 24,7 & 26,3 & 2,5 \\
\hline R $^{2}$ Ajustado & 0,64 & 0,54 & 0,72 & 0,60 & 0,64 & 0,68 & 0,05 \\
\hline
\end{tabular}

Un análisis más profundo de los estadísticos de distribución muestral demuestra que la localización de las plazas en alojamiento rural presenta bastantes inconvenientes, ya que tan solo se encuentran en la mitad de los núcleos analizados, algo que supondría cierta limitación en un análisis estadístico ordinario. No obstante, cuando se recurre al análisis territorial, se comprueba que existen zonas relativamente homogéneas sobre las que se 
concentra el mayor volumen de plazas, corroborando la primera ley de Tobler, según la cual todo está relacionado con el resto, si bien las cosas cercanas están más relacionadas que las distantes (Tobler, 1970).

Estos condicionantes pueden limitar que los porcentajes de explicación de la varianza no sean más elevados, pese a recurrir a la estadística espacial. De igual modo, se puede destacar otra posible causa, como es la apuesta por la implantación de alojamientos rurales en aquellos espacios en los que el potencial turístico no era idóneo.

Para establecer el grado de adaptación entre el volumen de plazas en alojamientos rurales y los valores que le podrían corresponder en función de sus atractivos, se propone la creación de una clasificación de 3 grupos bien delimitados mediante el grado de ajuste obtenido entre las plazas observadas y las estimadas por el modelo (Figura 6). En concreto, se establecen los siguientes:

- Núcleos con oferta de alojamientos rurales ajustada (residuales inferiores a \pm 25 y con presencia de alojamientos).

- Núcleos con oferta de alojamientos rurales saturada (residuales superiores a 25).

- Núcleos con oferta de alojamientos rurales insuficiente (sin plazas y con predicciones de más de 50 plazas).

\section{Figura 6 \\ AJUSTE ENTRE PLAZAS OBSERVADAS Y PREDICHAS SEGÚN GWR}

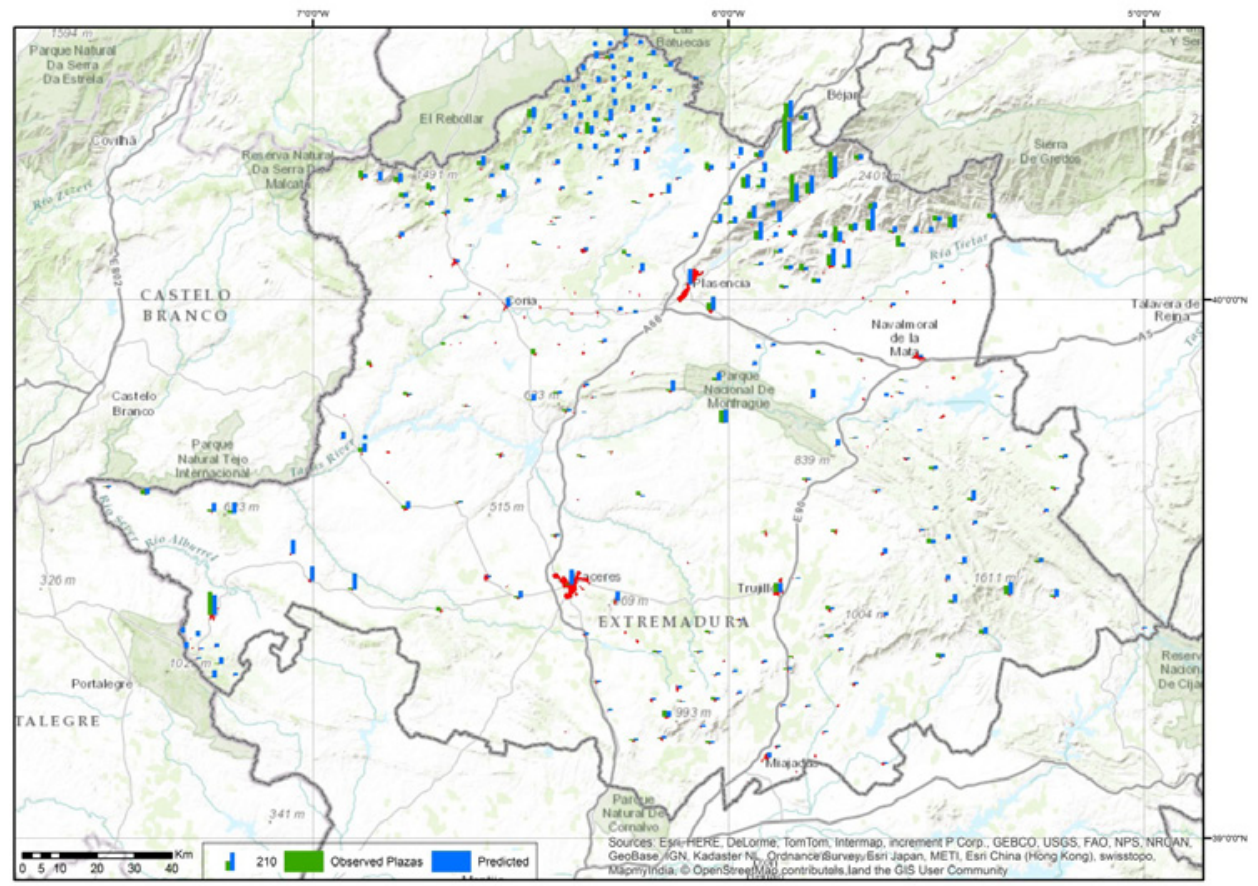




\subsection{Oferta de alojamientos rurales ajustada}

Esta categoría de oferta ajustada a los valores calculados por el modelo representa a todos aquellos núcleos en los que el residuo fluctúa entre \pm 25 plazas de alojamiento. Son 138 entidades que se aglutinan en 46 términos municipales.

La adaptación entre el volumen de plazas existente y el valor que predice la regresión es asumible a nuestro juicio, si bien se detectan dos tipos de núcleos diferenciados:

- Por una parte, los que no poseen alojamiento rural (71 núcleos), en los que el valor predicho es variable en el rango propuesto, si bien resulta más interesante en las entidades donde el valor predicho supera las 20 plazas y no disponen de ninguna en la realidad. Normalmente se trata de núcleos que no disponen de un potencial elevado, ya que mayoritariamente el mismo se situaría en la categoría "bajo".

\section{Figura 7}

\section{ZONAS CON OFERTA DE ALOJAMIENTOS RURALES ADAPTADA A SU POTENCIAL (EQUILIBRIO)}

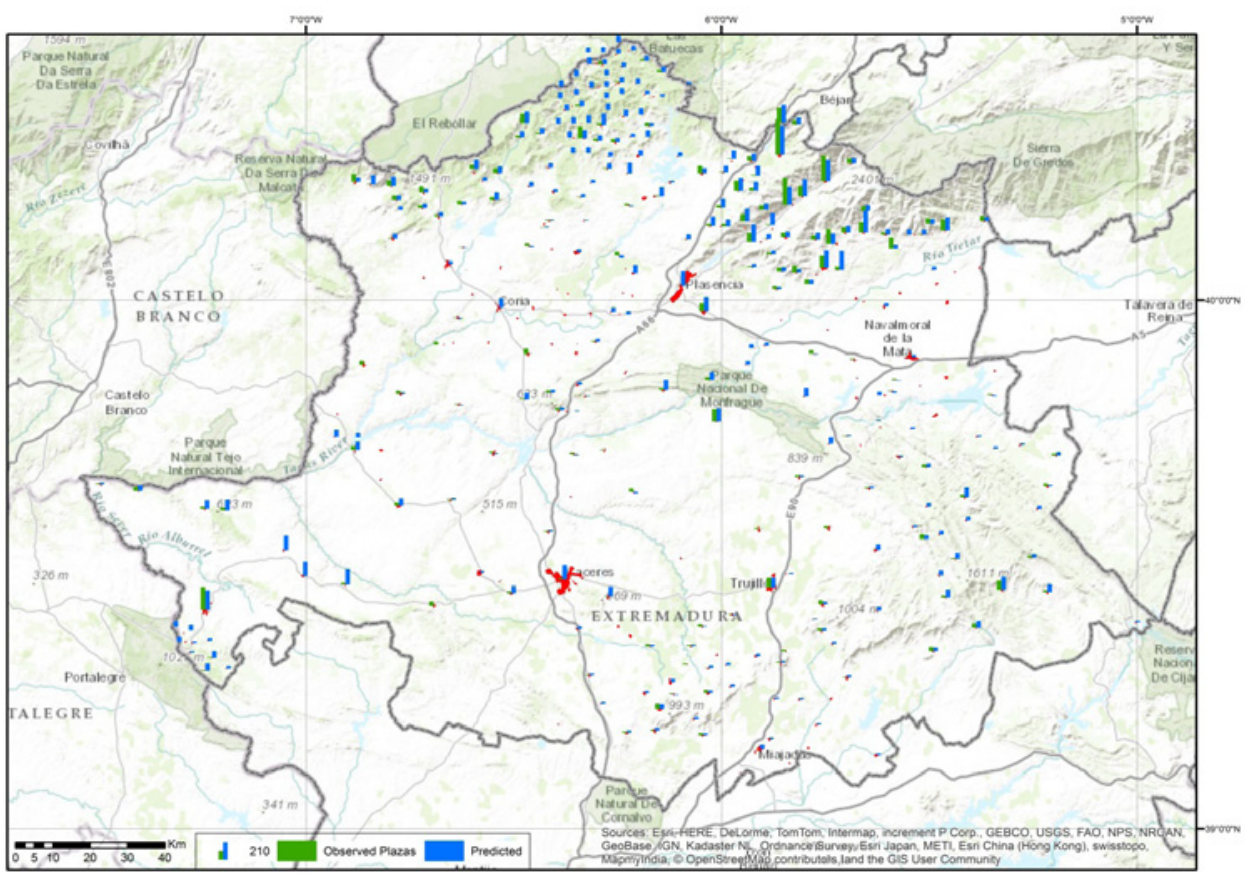

- Por otra, existen 67 entidades que sí disponen de alojamiento rural, en las que predominan valores predichos superiores a los existentes, siempre dentro del margen de error que se considera asumible. Entre ellos destacan algunos núcleos en los que el volumen de plazas existente es claramente inferior al predicho, como sucede 
en Cadalso, Rebollar, Casar de Palomero, Casares de Hurdes, Hoyos, Barrado, Gargüera, etc. Se observa así que en todos ellos, al menos tendría cabida un alojamiento rural (tipo casa rural) en los que, según los Decretos (120/98, 4/2000, $87 / 2007,204 / 2012$ ) que regulan este tipo de oferta, se pueden ofertar hasta 16 plazas. En este grupo se encuentran núcleos que disponen de un potencial "moderado" debido a que poseen algún atractor turístico interesante para la demanda, aunque no todos.

A pesar de todo, conviene recordar que el potencial turístico en buena parte de ellos puede resultar insuficiente para promover la implantación de alojamientos rurales, por lo que se cree necesario conocer qué potencial turístico tienen para detectar así si puede ser factible la instalación de un establecimiento. El resultado obtenido para las entidades que carecen de alojamiento aunque el modelo predice de 19 a 25 plazas no deja lugar a dudas, ya que en la mayor parte de los casos el potencial es bajo o, en el mejor de los casos, moderado.

\section{Tabla 7}

NÚCLEOS QUE PUEDEN ADMITIR ALGÚN ALOJAMIENTO RURAL

\begin{tabular}{|l|c|c|c|c|c|}
\hline Núcleo & $\begin{array}{c}\text { Plazas } \\
\text { Observadas }\end{array}$ & $\begin{array}{c}\text { Plazas } \\
\text { Predichas }\end{array}$ & Residuos & Potencial * & Equivalencia* \\
\hline Abertura & 0 & 19 & -19 & 12,4 & BAJO \\
\hline Arroyo de la Luz & 0 & 21 & -21 & 10,1 & BAJO \\
\hline Campillo de Deleitosa & 0 & 20 & -20 & 15,4 & MODERADO \\
\hline Casas de Don Antonio & 0 & 22 & -22 & 11,5 & BAJO \\
\hline Cíjara & 0 & 20 & -20 & 13,8 & BAJO \\
\hline Conquista de la Sierra & 0 & 24 & -24 & 13,5 & BAJO \\
\hline El Pino & 0 & 19 & -19 & 14 & BAJO \\
\hline Escurial & 0 & 18 & -18 & 10,6 & BAJO \\
\hline Garvín & 0 & 23 & -23 & 13 & BAJO \\
\hline Las Huertas de Cansas & 0 & 18 & -18 & 14 & BAJO \\
\hline Poblado Gabriel y Galán & 0 & 20 & -20 & 15,4 & MODERADO \\
\hline Retamosa de Cabañas & 0 & 22 & -22 & 16,7 & MODERADO \\
\hline Valdemorales & 0 & 23 & -24 & 11,9 & BAJO \\
\hline Villamesías & 0 & 20 & -20 & 12,4 & BAJO \\
\hline
\end{tabular}

* Datos obtenidos de Sánchez et al. (2013)

La localización de estos núcleos coincide mayoritariamente con la periferia de zonas de mayor atractivo, concentrándose en el entorno de la Sierra de Villuercas o en las últimas estribaciones de la Sierra de Montánchez. Esto implica una dificultad añadida a la hora de implantar cualquier tipo de alojamiento con vocación rural, ya que existen en otros núcleos cercanos, siendo éstos los que reúnen mayor potencial turístico. 


\subsection{Oferta de alojamientos rurales saturada}

La sobreoferta se produce cuando los valores que estima el modelo son inferiores a los calculados mediante nuestro modelo de regresión. En este caso se ha optado por incluir los núcleos que tienen un residual superior a 25, es decir, se incluyen las entidades de población en los que la oferta existente es superior a la estimada.

\section{Figura 8}

\section{ZONAS CON OFERTA DE ALOJAMIENTOS RURALES INADAPTADA A SU POTENCIAL (POSIBLE EXCESO)}

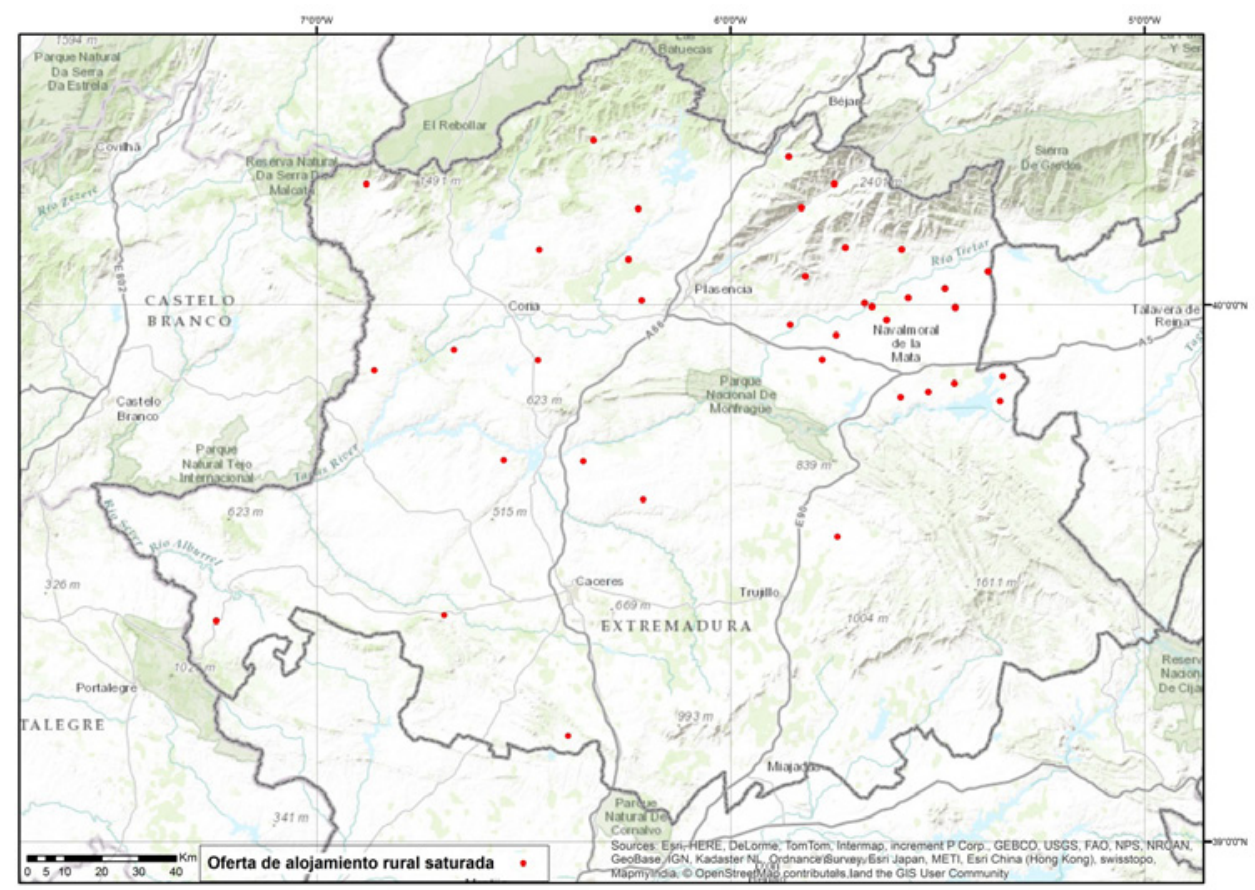

En esta categoría se integran 38 núcleos de población agrupados en 36 términos municipales, de los que 15 carecen de alojamiento, algo que implica una adaptación a la poca potencialidad del territorio. El resto de entidades oferta plazas en alojamiento rural en forma variable.

Se trata, en líneas generales, de entidades en las que el volumen de plazas es considerable si se tiene en cuenta el potencial turístico disponible, estando encabezados por núcleos como Guijo de Coria, Toril o Valdeobispo. Son núcleos peculiares pues en ellos está presente la oferta de alojamientos, por lo que es posible que precisen algún tipo de actuación con el fin de ganar competitividad. Pese a ello, los núcleos en los que se detecta un mayor volumen de oferta en alojamientos rurales suelen coincidir con aquéllos en 
los que el potencial turístico resulta más elevado, como sucede en los casos de Hervás, Navaconcejo, Jerte, etc. En estos últimos, la situación no resulta tan preocupante, ya que cuentan con la ventaja de ser entidades en las que la actividad está plenamente asentada y se encuentran localizados en la zona norte de la provincia, la más interesante para la práctica del turismo rural.

Tabla 8

VALORES REALES SUPERIORES

A LOS PREDICHOS POR EL MODELO

\begin{tabular}{|c|c|c|c|c|c|}
\hline Núcleo & $\begin{array}{c}\text { Plazas } \\
\text { Observadas }\end{array}$ & $\begin{array}{c}\text { Plazas } \\
\text { Predichas }\end{array}$ & Residuos & Potencial* & Equivalencia* \\
\hline Acebo & 69 & 43 & 26 & 19,8 & MODERADO \\
\hline Aliseda & 50 & 1 & 49 & 11,9 & BAJO \\
\hline Berrocalejo & 12 & -15 & 27 & 11,1 & BAJO \\
\hline Cachorrilla & 30 & -3 & 33 & 14 & BAJO \\
\hline Casas de Belvís & 8 & -21 & 29 & 16,4 & MODERADO \\
\hline Cuacos de Yuste & 172 & 122 & 51 & 19,5 & MODERADO \\
\hline Garrovillas de Alconétar & 17 & -11 & 28 & 11,9 & BAJO \\
\hline Guijo de Coria & 31 & -16 & 47 & 12,8 & BAJO \\
\hline Hervás & 412 & 337 & 75 & 22,6 & ALTO \\
\hline Hinojal & 18 & -15 & 33 & 12,8 & BAJO \\
\hline Jerte & 291 & 243 & 48 & 21,6 & ALTO \\
\hline Monroy & 36 & 4 & 32 & 15,4 & MODERADO \\
\hline Navaconcejo & 313 & 208 & 105 & 20,8 & ALTO \\
\hline Pasarón de la Vera & 78 & 42 & 36 & 17,7 & MODERADO \\
\hline Pinofranqueado & 139 & 94 & 45 & 20,3 & ALTO \\
\hline Robledillo de La Vera & 132 & 48 & 84 & 17,6 & MODERADO \\
\hline Santibáñez el Bajo & 20 & -14 & 34 & 13,5 & BAJO \\
\hline Toril & 40 & -12 & 52 & 14,8 & BAJO \\
\hline Torrecillas de la Tiesa & 27 & -1 & 28 & 13,7 & BAJO \\
\hline Torrejoncillo & 63 & 0 & 63 & 15,6 & MODERADO \\
\hline Valdeobispo & 42 & -30 & 72 & 11,5 & BAJO \\
\hline Valencia de Alcántara & 264 & 225 & 39 & 16,1 & MODERADO \\
\hline Valverde del Fresno & 87 & 48 & 39 & 18,4 & MODERADO \\
\hline Zarza la Mayor & 47 & -3 & 50 & 15,6 & MODERADO \\
\hline
\end{tabular}

* Datos obtenidos de Sánchez et al. (2013) 


\subsection{Oferta de alojamientos rurales insuficiente}

En esta categoría se engloban los núcleos que tienen una oferta insuficiente, ya que el modelo estima un volumen de plazas sensiblemente superior a las existentes. Esto se debe a que aglutinan en buena parte de los casos un potencial moderado. Se compone de 100 entidades de población integradas en 45 términos municipales, de lo que se deduce el gran potencial para el desarrollo de esta actividad que tienen las entidades menores, concentradas muchas de ellas en la comarca de Hurdes, o capitales de municipio que se encuentran en la zona de influencia del Parque Nacional de Monfragüe, del Parque Natural de Tajo Internacional o del Geoparque de Villuercas-Ibores-La Jara. Si a ello se añade que buena parte de las entidades no tienen ningún alojamiento rural, es posible afirmar que el crecimiento de la actividad puede ser una realidad (Tabla 10).

\section{Figura 9}

\section{ZONAS CON OFERTA DE ALOJAMIENTOS RURALES INADAPTADA A SU POTENCIAL (DÉFICIT)}

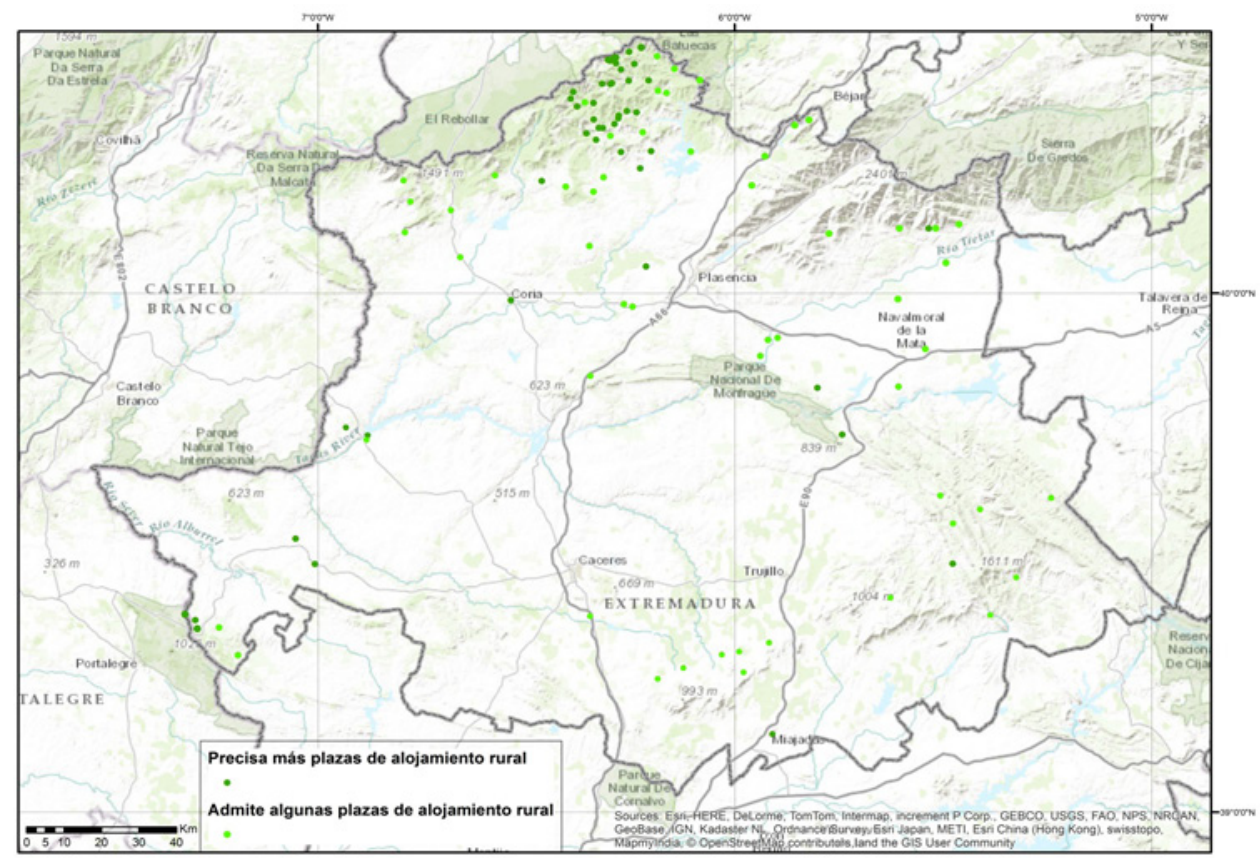

Pese a ello, cabe recordar que en buena parte de estos núcleos el potencial turístico es moderado y bajo, a lo que se debe añadir la existencia de una oferta rural en el entorno próximo, algo que puede suponer una limitación a la hora de implantar un nuevo alojamiento. Dada la cantidad de núcleos presentes en esta categoría, se han expuesto únicamente los casos que disponen de potencial caracterizado como óptimo o bueno. 
Tabla 9

ENTIDADES CON POTENCIAL PARA LA IMPLANTACIÓN DE ALOJAMIENTOS RURALES

\begin{tabular}{|c|c|c|c|c|c|}
\hline Núcleo & $\begin{array}{c}\text { Plazas } \\
\text { Observadas }\end{array}$ & $\begin{array}{c}\text { Plazas } \\
\text { Predichas }\end{array}$ & Residuos & Potencial* & Equivalencia* \\
\hline Alcántara & 43 & 92 & -49 & 25,89 & BUENO \\
\hline Aldeanueva del Camino & 55 & 83 & -28 & 29,16 & BUENO \\
\hline Arrolobos & 0 & 29 & -29 & 25,14 & BUENO \\
\hline Asegur & 0 & 61 & -61 & 25,31 & BUENO \\
\hline Azabal & 26 & 61 & -35 & 27,51 & BUENO \\
\hline Baños de Montemayor & 169 & 202 & -33 & 34,99 & OPTIMO \\
\hline Belvís de Monroy & 0 & 39 & -39 & 25,33 & BUENO \\
\hline Cañamero & 49 & 79 & -30 & 30,38 & OPTIMO \\
\hline Carcaboso & 0 & 96 & -96 & 26,19 & BUENO \\
\hline Casas de Miravete & 0 & 74 & -74 & 27,08 & BUENO \\
\hline Casas del Monte & 117 & 149 & -32 & 28,6 & BUENO \\
\hline Cerezal & 0 & 64 & -64 & 26,43 & BUENO \\
\hline Fragosa & 0 & 65 & -65 & 25,13 & BUENO \\
\hline Garganta (La) & 43 & 76 & -33 & 32,5 & OPTIMO \\
\hline Garganta la Olla & 51 & 82 & -31 & 32,18 & OPTIMO \\
\hline Guadalupe & 105 & 146 & -41 & 30,86 & OPTIMO \\
\hline Hernán Pérez & 0 & 52 & -52 & 25,33 & BUENO \\
\hline Ladrillar & 0 & 51 & -51 & 26,61 & BUENO \\
\hline Losar de la Vera & 26 & 65 & -39 & 32,1 & OPTIMO \\
\hline Mestas, Las & 24 & 62 & -38 & 26,3 & BUENO \\
\hline Moraleja & 0 & 32 & -32 & 25,68 & BUENO \\
\hline Navalmoral de la Mata & 0 & 28 & -28 & 25,06 & BUENO \\
\hline Perales del Puerto & 18 & 45 & -27 & 26,54 & BUENO \\
\hline Robledollano & 0 & 43 & -43 & 25,16 & BUENO \\
\hline San Gil & 0 & 37 & -37 & 25,21 & BUENO \\
\hline San Martín de Trevejo & 66 & 110 & -44 & 29,24 & BUENO \\
\hline Sauceda & 0 & 73 & -73 & 25,38 & BUENO \\
\hline Serrejón & 0 & 98 & -98 & 25,26 & BUENO \\
\hline Talaveruela de la Vera & 10 & 58 & -48 & 26,53 & BUENO \\
\hline Torre de Don Miguel & 0 & 42 & -42 & 25,26 & BUENO \\
\hline Urdimalas & 0 & 43 & -43 & 25,61 & BUENO \\
\hline Vegas de Coria & 0 & 41 & -41 & 25,3 & BUENO \\
\hline Viandar de la Vera & 0 & 58 & -58 & 27,58 & BUENO \\
\hline Villanueva de la Vera & 130 & 156 & -26 & 30,8 & OPTIMO \\
\hline
\end{tabular}

* Datos obtenidos de Sánchez et al. (2013) 


\section{CONCLUSIONES}

Cualquier aplicación de estadística multivariada exige el cumplimiento de una serie de requisitos previos, entre los que destaca la depuración de variables explicativas, de tal forma que se elimine cualquier indicio de colinealidad. Esto cobra especial relevancia en la regresión geográficamente ponderada, ya que puede resultar tan grave que inutilice los modelos predictivos generados. Por este motivo, en este artículo se ha propuesto una secuencia lógica encaminada a la reducción o eliminación de este grave problema que aparece cuando se consideran predictores fuertemente correlacionados, siendo esta aportación metodológica una de las principales novedades del mismo.

Entre las principales conclusiones que se extraen, es posible destacar las siguientes, referidas tanto a las técnicas utilizadas como a sus resultados.

En primer lugar, se concluye que es preciso depurar cualquier modelo de regresión de tal forma que las variables incluidas no presenten fuertes correlaciones, incluso a cambio de perder representatividad en la explicación de la varianza. Dentro de las diferentes formas de depurar las variables correlacionadas, pese a que no existe un estándar, se ha apostado como paso previo por la aplicación de una regresión de mínimos cuadrados ordinarios. Según esta técnica, cuando se supera un umbral crítico en el factor de inflación de la varianza (VIF), existe un grave problema de colinealidad entre las variables predictoras, por lo que se hace imprescindible eliminar todas aquéllas fuertemente correlacionadas. Esto, a la postre, posibilita obtener un modelo en el que la relación entre las variables independientes sea lo más baja posible, algo que también se corrobora mediante la aplicación de una correlación lineal entre dichas variables.

En segundo lugar, para comprobar la bondad del modelo se considera necesaria la aplicación de una autocorrelación espacial mediante la formulación I de Moran sobre los residuales obtenidos mediante la regresión de mínimos cuadrados ordinarios, cuyo objetivo es determinar que estos desajustes entre los valores predichos y observados tienen una distribución aleatoria y no concentrada sobre el territorio, algo que podría interpretarse como la omisión de algún predictor localizado en la zona donde se concentran estos residuos.

En tercer lugar, los resultados que ofrece la regresión permiten analizar la adecuación del modelo a cada una de las entidades analizadas, ya que se desarrolla una ecuación individualizada, algo que combinado con el factor de condición nos muestra el grado de ajuste y la confianza que tiene el modelo en cada espacio. Asimismo, si se apuesta por la comparación entre los valores observados y calculados por el modelo, es posible determinar diferentes grupos de núcleos en los que la oferta de alojamiento rural presenta una casuística diferente respecto de los valores predichos por el modelo.

En cuarto lugar, partiendo del grado de desajuste entre los valores reales y calculados, es posible deducir un grupo clave de núcleos en los que la oferta real puede estar sobredimensionada máxime si se tiene en cuenta el potencial turístico que tienen y, mutatis mutandis, ésta será insuficiente cuando los valores predichos sean muy superiores a los reales, siempre considerando el potencial para el desarrollo del turismo rural que tiene cada núcleo.

Por último, se destaca que la localización de la oferta de alojamientos rurales sigue normalmente las pautas que marca las aptitudes del territorio para la práctica del turismo 
rural. No obstante, se han detectado ciertas anomalías consistentes en núcleos que disponen de poco potencial turístico para sustentar con garantías de éxito una oferta de este tipo, sobre todo cuando se carece de los principales atractivos que demanda el turista, algo que sucede en comarcas como el Campo Arañuelo. También se observan núcleos que, pese a disponer de potencial turístico elevado, cuentan con un volumen de plazas muy abundante, posiblemente más de las que puede mantener su atractivo. Pese a esta circunstancia, también son numerosos los núcleos con una oferta ajustada al potencial disponible, distribuidas por todas las zonas que tienen cierto grado de atractivo turístico. Al mismo tiempo, aparecen áreas deficitarias de oferta que se concentran en zonas muy concretas y bien localizadas en el territorio, como sucede en la comarca de Hurdes, así como el entorno del parque Nacional de Monfragüe. Además se da la circunstancia de que en la periferia de estas zonas y otras con gran atractivo, se localizan núcleos que podrían sustentar aún más oferta.

\section{BIBLIOGRAFÍA}

ÁLVAREZ CUERVO, R. y LENO CERRO, F. (1986): Estudio para la valoración económica y situación estructural del mercado turístico riojano. Madrid, Instituto de Estudios Turísticos- Gobierno de la Rioja.

BELSLEY, D.A. (1980): «On the efficient computation of the nonlinear full-information maximum-likelihood estimator», Journal of Econometrics, vol. 14, n 2, pp. 203-225.

BELSLEY, D. A. (1982): «Assessing the presence of harmful collinearity and other forms of weak data through a test for signal-to-noise», Journal of Econometrics, vol. 20, $\mathrm{n}^{\circ}$ 2, pp. 211-253.

BOTE GÓMEZ, V. (1990): Planificación económica del turismo. De una estrategia masiva a una artesanal. México, Trillas.

BRIDA, J.G.; RIAÑO, E. y ZAPATA, S. (2012): «Percepciones de los residentes acerca de los impactos del turismo de cruceros en la comunidad: un análisis factorial y de clústeres», Cuadernos de Turismo, n 29, pp. 79-107.

BRUNSDON, C.; FOTHERINGAM, S. y CHARLTON, M. (2002): «Geographically Weighted Local Statistics Applied to Binary Data», Lecture Notes in Computer Science, $\mathrm{n}^{\mathrm{o}} 2478$, pp. 38-50.

BURNHAM, K.P. y ANDERSON, D.R. (2002): Model selection and multimodel inference: a practical information-theoretic approach. New York, Springer.

CABALLERO DÍAZ, F. F. (2011): Selección de modelos mediante criterios de información en análisisfactorial. Aspectos teóricos y computacionales.(Tesis Doctoral). 10 de febrero de 2015. Disponible en http://digibug.ugr.es/bitstream/10481/19428/1/19964808.pdf

CÁNOVES, G.; VILLARINO, M.; PRIESTLEY, G. y BLANCO, A. (2002): «Rural tourism in Spain: an analysis of recent evolution», Geoforum, no 35, pp. 755-769.

CASAS SÁNCHEZ, J.M. y SANTOS PEÑAS, J. (2002): Introducción a la estadística para la economía y administración de empresas. Madrid, Editorial Centro de Estudios Ramón Areces.

CELEMÍN, J.P. (2009): «Autocorrelación espacial e indicadores locales de asociación espacial: Importancia, estructura y aplicación», Revista Univesitaria de Geografía, vol. $18, \mathrm{n}^{\mathrm{o}} 1$, pp. 11-31. 
CHARLTON, M. y BRUNSDON, C. (1997): «Two Techniques for Exploring Non-stationarity in Geographical Data», Journal of Geographical Systems, vol. 4, nº 1, pp. 59-82.

CHEN, V.J.; DENG, W.S.; YANG, T.C. y MATTHEWS, S. . (2012): «Geographically weighted quantile regression (GWQR): An application to US mortality data», Geographical Analysis, n ${ }^{\circ}$ 44, pp. 132-150.

DUQUE, J.C.; VELÁSQUEZ, H. y AGUDELO, J. (2011): «Infraestructura pública y precios de vivienda: una aplicación de regresión geográficamente ponderada en el contexto de precios hedónicos», Ecos de Economía, n 33, pp. 95-122.

FOTHERINGHAM, S. y BRUNSDON, C. (1999): «Local Forms of Spatial Analysis», Geographical Analysis, $\mathrm{n}^{\circ} 31$, pp. 340-358.

GELFAND, A.; KIM, H-J.; SIRMANS, C.F. y BANERJEE, S. (2003): «Spatial Modeling with Spatially Varying Coefficient Processes», Journal of the American Statistical Association, vol. 98, n 462, pp. 387-396.

HALL, D. (2004): «Rural Tourism Development in Southeastern Europe: Transition and the Search for Sustainability», International Journal of Tourism Research, vol. 6, $\mathrm{n}^{\circ}$ 3, pp. 165-176.

HERNÁNDEZ LÓPEZ, M. (2002): Algoritmos genéticos y predicción de la composición de la demanda turística. (Tesis Doctoral). Disponible en ftp://tesis.bbtk.ull.es/ ccssyhum/cs120.pdf

HUANG, Y. y LEUNG, Y. (2002): «Analysis Regional Industrialisation in Jiangsu Province Using Geographically Weighted Regression», Journal of Geographical Systems, vol. $4, n^{\circ} 2$, pp. 233-249.

HURVICH, C.M. y TSAI, Ch-L. (1989): «Regression and time series model selection in small samples», Biometrika, vol. 76, n 2, pp. 297-307.

IGLESIAS I MILLAN, J. A. (2005): Técnicas de Investigación aplicadas al sector turístico. Madrid, Editorial Síntesis.

JONES, C. (1995): «Time Series Tests of Endogenous Growth Models», The Quarterly Journal of Economics, vol. 110, n 2, pp. 495-525.

LENO CERRO, F. (1993): Técnicas de evaluación del potencial turístico. Madrid, Ministerio de Industria, Comercio y Turismo.

LLOYD, C.D. y SHUTTLEWORTH, I. (2005): «Analysing commuting using local regression technique: scale, sensitivity and geographical patterning», Environment and Planning $A$, vol. $37 n^{\circ} 1$, pp. 81-103.

LONGLEY, P. y TOBÓN, C. (2004): «Spatial Dependence and Heterogeneity in Patterns of Hardship: An Intra-Urban Analysis», Annals of the Association of American Geographers, vol. 94, pp. 503-519.

LÓPEZ FERNÁNDEZ, M.C.; SERRANO BEDIA, A.M. y GÓMEZ LÓPEZ, R. (2007): «Caracterización del comportamiento innovador de las empresas hoteleras españolas», Revista de Análisis Turístico, no 4, pp. 22-35.

LÓPEZ OLIVARES, D. (2003): «La evaluación de los recursos territoriales turísticos de las comarcas del interior castellonense (Comunidad Valenciana)», Investigaciones geográficas, $\mathrm{n}^{\circ}$ 32, pp. 11-135. 
LÓPEZ ONTIVEROS, A. y MULERO MENDIGORRI, A. (1999): «Síntesis de la investigación reciente sobre recreación rural en España (1960-1995)», Agricultura y Sociedad, $\mathrm{n}^{\circ} 83$, pp. 77-116.

MENNIS, J.L. y JORDAN, L. (2005): «The distribution of environmental equity: exploring spatial nonstationarity in multivariate models of air toxic releases», Annals of the Association of American Geographers, $\mathrm{n}^{\circ}$ 95, pp. 249-268.

POWELL, J.L. (1991): «Estimation of Monotonic Regression Models under Quantile Regressions», en Nonparametric and Semiparametric Models in Econometrics, ed. W. Barnett et al. Cambridge, Cambridge University Press, pp. 357-386

RACHUDEL, W.J. (1971): Multicollinearity once again. Cambridge, Harvad Institute of Economic Research.

RENGIFO GALLEGO, J.I.; SÁNCHEZ RIVERO, M. y SÁNCHEZ MARTÍN, J.M. (2013): «Análisis del desarrollo del turismo rural en la provincia de Cáceres en los inicios del siglo XXI». PASOS. Revista de Turismo y Patrimonio Cultural, vol. 11, pp. 615-630.

RONQUILLO MELCIO, A. (1997): Estadística aplicada al sector turístico. Técnicas cuantitativas y cualitativas de análisis turístico. Madrid, Editorial Centro de Estudios Ramón Areces.

SAN MARTÍN GUTIÉRREZ, H.; RODRÍGUEZ DEL BOSQUE RODRÍGUEZ, I.A. y VÁZZUEZ CASIELLES, R. (2006): «Análisis de la imagen en turismo mediante técnicas estructuradas y no estructuradas: Implicaciones competitivas para los destinos turísticos», Revista Asturiana de Economía, no 35, pp. 69-91.

SÁNCHEZ MARTÍN, J.M. (1995): «La matriz de correlación lineal en Climatología. Los riesgos interpretativos: Su reducción o eliminación», Estudios Geográficos, nº 219 , pp. 411-433.

SÁNCHEZ MARTÍN, J.M. (2009): «El Sistema de Información Geográfica como herramienta de planificación turística. Una aplicación para la localización idónea de alojamientos rurales en la provincia de Cáceres», Estudios Turísticos, n 182, pp. 71-94.

SÁNCHEZ MARTÍN, J. M.; GURRÍA GASCÓN, J. L.; LECO BERROCAL, F. y PÉREZ MARTÍN, M. N. (2001): «SIG para el desarrollo turístico en los espacios rurales de Extremadura», Revista Estudios Geográficos, vol. 62, n²43, pp. 335-368.

SÁNCHEZ MARTÍN, J.M.; SÁNCHEZ RIVERO, M. y RENGIFO GALLEGO, J.I. (2013): «La evaluación del potencial para el desarrollo del turismo rural. Aplicación metodológica sobre la provincia de Cáceres», Geofocus, vol. 13, nº 1, pp. 99-130.

SÁNCHEZ-PEÑA, L.L. (2012): «Alcances y límites de los métodos de análisis espacial para el estudio de la pobreza urbana», Papeles de Población, vol. 18, n 272, pp. 147-179.

SÁNCHEZ RIVERO, M. (2008): «Análisis espacial de datos y turismo: nuevas técnicas para el análisis turístico. Una aplicación al caso extremeño», Revista de Estudios Empresariales. Segunda Época, $\mathrm{n}^{\circ}$ 2, pp. 48-66.

SÁNCHEZ RIVERO, M., SÁNCHEZ MARTÍN, J.M. y RENGIFO GALLEGO, J.I. (2014): «Methodological approach for assessing the potential of a rural tourism destination: An application in the province of Cáceres (Spain)», Current issues in tourism. 15 de diciembre de 2014. Disponible en http://www.tandfonline.com/doi/abs/10.1080 /13683500.2014.978745. 
SHARPLEY, R. (2002): «Rural tourism and the challenge of tourism diversification: the case of Cyprus», Tourism Management, vol. 23, $\mathrm{n}^{\circ} 3$, pp. 233-244.

TAYLOR, J.W. (2008): «Using Exponnentially Weighted Quantile Regression to Estimate Value at Risk and Expected Shortfall», Journal of Financial Econometrics, vol. 6, $\mathrm{n}^{\circ}$ 3, pp. 382-406.

TOBLER, W.R. (1970): «A computer model simulation of urban growth in the Detroit region», Economic Geography, vol. 6, $\mathrm{n}^{\circ}$ 2, pp. 234-240. 2 de febrero de 2015. Disponible en http://www.jstor.org/stable/143141

URIEL JIMÉNEZ, E. (1997): Econometría: El Modelo Lineal. Madrid: Editorial Alfa Centauro.

WHEELER, D.C. \& TIEFELSDORD, M.R. (2005): «Multicollinearity and Correlation among Local Regression Coefficients in Geographically Weighted Regression», Journal of Geographical Systems, vol. 7, n 2, pp. 161-187. 